\title{
Contents of Volume 135 (2003)
}

Volume 135 No. 1, January (I) 2003

Jean-Marc Schlenker and Rabah Souam: Higher Schläfli Formulas and Applications

Tom Graber and Ravi Vakil: Hodge Integrals and Hurwitz Numbers via Virtual Localization

Paul Broussous: Un revêtement de l'arbre de $\mathrm{GL}_{2}$ d'un corps local (A Covering of the $\mathrm{GL}_{2}$ Tree of a Local Field)

Kimitoshi Ukai: $b$-Functions of Prehomogeneous Vector Spaces of Dynkin-Kostant Type for Exceptional Groups

Vladimir Tchernov: Isomorphism of the Groups of Vassiliev Invariants of Legendrian and Pseudo-Legendrian Knots

\section{Volume 135 No. 2, January (II) 2003}

Claus Mokler: A Formal Chevalley Restriction Theorem for Kac-Moody Groups

William Banks, Daniel Bump and Daniel Lieman: Whittaker-Fourier Coefficients of Metaplectic Eisenstein Series

A. A. Du Plessis and C. T. C.Wall: Generic Projections in the Semi-nice Dimensions

Dinakar Ramakrishnan and Song Wang: On the Exceptional Zeros of Rankin-Selberg $L$-Functions

Volume 135 No. 3, February 2003

Paul Bressler and Valery A. Lunts: Intersection Cohomology on Nonrational Polytopes

V. Apostolov, D. M. J. Calderbank and P. Gauduchon: The Geometry of Weakly Self-dual Kähler Surfaces 
Christina Birkenhake and Pol Vanhaecke: The Vanishing of the Theta Function in the KP Direction: A Geometric Approach

$323-330$

Gang Yu: Rank 0 Quadratic Twists of a Family of Elliptic Curves

$331-356$

Author Index, Volume 135 (2003) 